\title{
Dermatology
}

Published online: November 13, 2008

Dermatology 2009;218:75-78

DOI: $\underline{10.1159 / 000174102}$

\section{Cutaneous T Cell Pseudolymphoma at the Site of a Semipermanent Lip-Liner Tattoo}

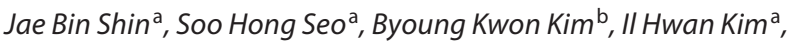 Sang Wook Son ${ }^{\text {a }}$}

Departments of ${ }^{\mathrm{a}}$ Dermatology and ${ }^{\mathrm{b}}$ Occupational and Environmental Medicine, Korea University College of Medicine, Seoul, Korea

Key Words

Pseudolymphoma $\cdot$ Semipermanent tattoo $\cdot$ Lip-liner tattoo

\section{Introduction}

Tattoos are becoming more popular not only as a form of body art, but also for cosmetic adornment. Complications, such as allergic reactions, infections and incidental tumorous lesions brought about by this fashionable insult are often neglected. Semipermanent tattoo cosmetic adornment is becoming increasingly popular in Western and East-Asian societies. We present a case of a pseudolymphoma localized to the site of a semipermanent lip-liner tattoo.

\section{Case Report}

A 46-year-old woman presented with a 1-year history of linear swelling on both lips, which had developed gradually after application of a semipermanent lip-liner tattoo. She denied any localized symptoms, such as pruritus or tenderness, and had no other specific illnesses.

The physical examination revealed red-colored and indurated swelling along the lines outlined with tattoo ink on both the upper and lower lip margins (fig. 1a). Laboratory findings, including a complete blood count, serum chemistry and peripheral blood smear, were all within normal limits.

Histopathologic examination of a skin biopsy specimen showed diffuse and dense cellular infiltration through the entire dermis (fig. 2a). The inflammatory infiltrate was mainly composed of lymphocytes, but also included macrophages in the upper dermis that occasionally contained pigment granules (fig. 3). There was no evidence of germinal center or lymphoid follicle formation. Immunohistochemistry showed that the majority of the infiltrated cells strongly expressed CD3 antigen (fig. 2b). The lymphocytes were CD68 negative, but the macrophages partially expressed CD68 (fig. 2c). CD20 and terminal deoxynuleotidyl transferase were negative (fig. 2d, e). Ki-67 had a low proliferation index (fig. 2f). A T cell receptor $\gamma$ gene rearrangement study was performed on paraffin-embedded skin and showed a polyclonal pattern.

We attempted treatment with a 595-nm pulsed dye laser, but there was no improvement after 4 sessions. Thereafter, the patient underwent intralesional triamcinolone injections at 2- or 3-week intervals for 4 months. The lesion responded gradually and marked clinical improvement was observed (fig. 1b). During a fol-

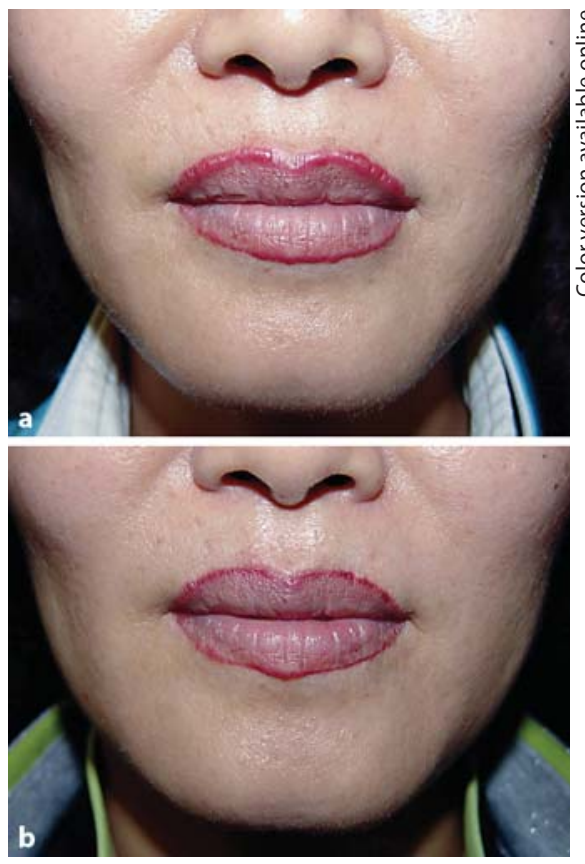

Fig. 1. Skin lesion before and after treatment. a Red-colored and indurated swelling along the lip-liner tattoo site of both lips. b Near-complete resolution after intralesional corticosteroid injections for 4 months.

\section{KARGER}

(C) 2008 S. Karger AG, Basel

Fax +41613061234 E-Mail karger@karger.ch www.karger.com 

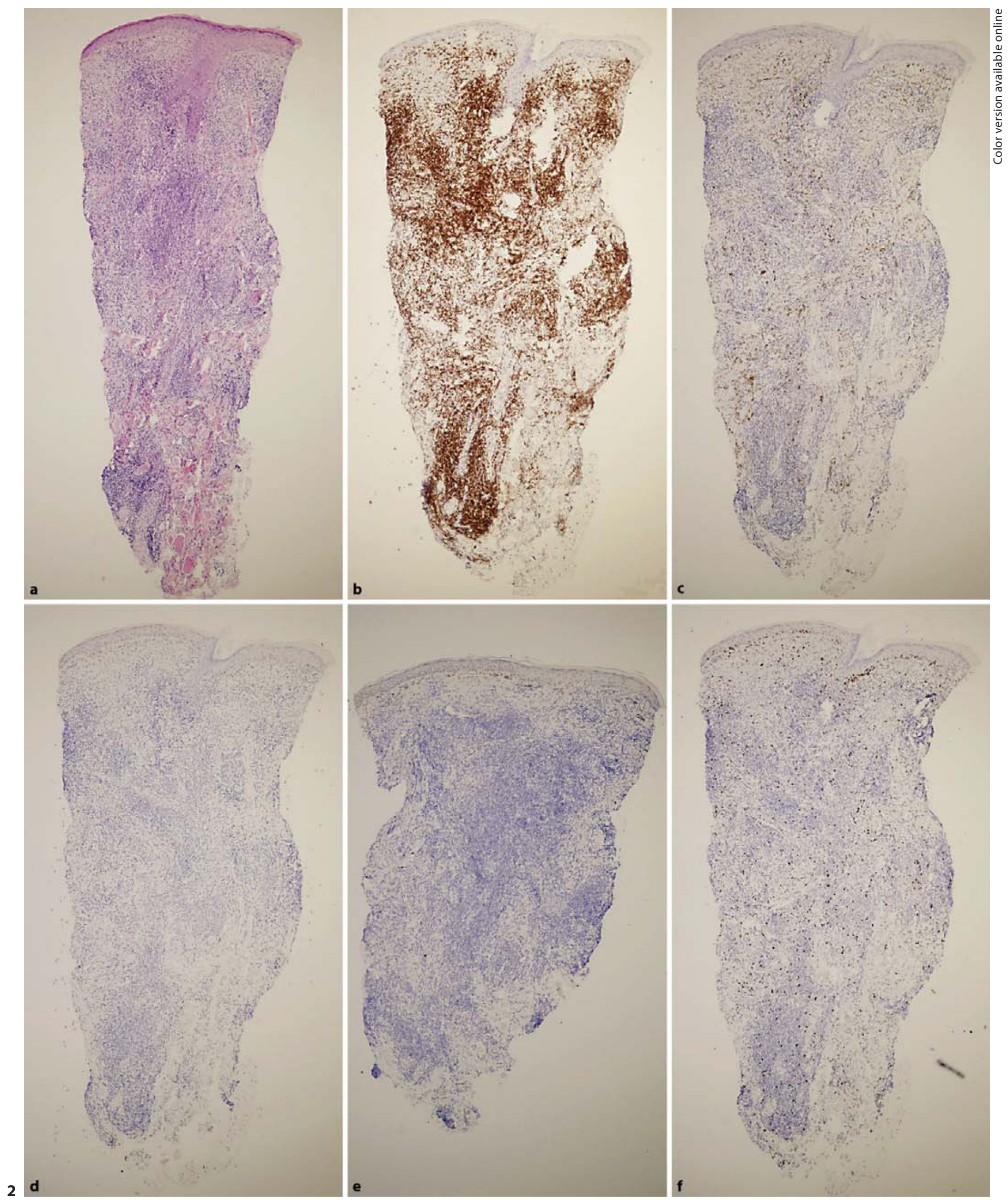
low-up period of 1 year, the patient showed partial recurrence of the previous lesion.

\section{Discussion}

A pseudolymphoma is defined as a process that simulates a lymphoma, primarily histologically, but sometimes clinically. At the time of diagnosis, pseudolymphomas appear to have a benign biologic behavior and do not satisfy the criteria for malignant lymphomas [1]. Tattoo-induced cutaneous pseudolymphomas generally belonged to cutaneous B cell pseudolymphomas $[1,2]$. However, tattoo-induced cutaneous $\mathrm{T}$ cell pseudolymphoma has been reported previously $[3,4]$. In our case, the main infiltrate also presented $\mathrm{T}$ cell predominant features. Therefore, adjustment or reclassification of cutaneous pseudolymphomas relating to tattoos is needed.

The pathogenesis of tattoo-induced pseudolymphomas is still unknown. Tattoo dyes contain various kinds of metal compounds and organic substances, such as azo dyes. These components have been suggested to be causative agents for delayed hypersensitivity or some malignancies [5-10]. Among these materials, allergic reactions to certain dyes, especially to red mercury-based dyes, are well known [5, 6]. In most cases, as in the current case, tattoo-induced cutaneous pseudolymphomas are also reported in the red portions of the tattoo [4].

We obtained a sample of the tattoo dye and analyzed the components. We used a furnace and cold-vapor method with an atomic absorption spectrophotometer (AAnalyst-800; Perkin Elmer, USA). These methods showed the presence of metal iron (12.677 $\mathrm{mg} / \mathrm{l})$, copper fumes $(1.867 \mathrm{mg} / \mathrm{l})$, metal manganese $(0.289 \mathrm{mg} / \mathrm{l})$ and metal cobalt $(0.013 \mathrm{mg} / \mathrm{l})$. Other metals, such as mercury, cadmium and titanium, were not detected by our methods. An organic mercury compound had been thought to be the main cause of red tattoo reactions [5], but our methods could not detect one. Abnormal tattoo reactions in the absence of mercury have also been reported [11]. We considered components other than mercury, the detected metals in this case, or an undetected dye to be the cause of the pseudolymphoma.

The time of onset of pseudolymphomas after tattooing ranges from a few months to 12 years [12]. Pseudolymphomas present as nodular growths or swellings. Histopathologic examination of pseudolymphomas reveals a diffuse and dense infiltrate in the entire dermis. The infiltrate is mainly composed of small lymphocytes and macrophages, with some eosinophils and few plasma cells [13]. In the case of B cell dominance, a germinal center or lymphoid follicle may be seen. Immunohistochemical techniques and polymerase chain reaction for $\mathrm{T}$ cell receptor or immuno-

Fig. 2. Punch biopsy specimen of the lesion. a Diffuse and dense cellular infiltrations through the entire dermis. HE. b The majority of infiltrated cells showed strong expression of CD3 antigen. Anti-CD3 stain. c Anti-CD68 stain was negative for the majority of lymphocytes, but was partially expressed by macrophages. Anti-CD20 (d) and terminal deoxynuleotidyl transferase stains (e) were negative. f Anti-Ki-67 stain showed a low proliferation index. In all cases, original magnification was $\times 40$.

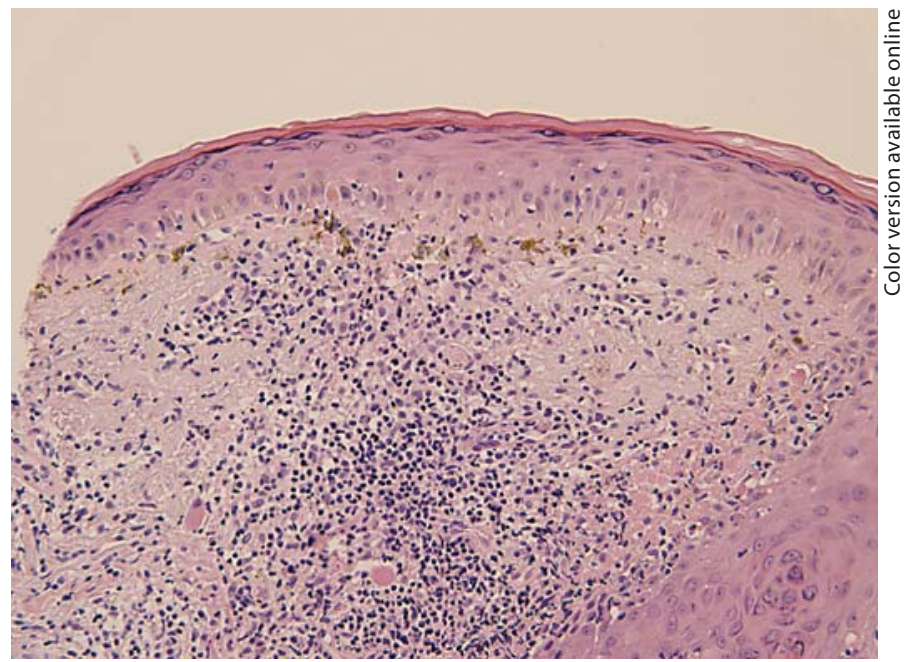

Fig. 3. The inflammatory infiltrate was mainly composed of lymphocytes, but also included macrophages that occasionally contained pigment granules in the upper dermis. HE. $\times 200$.

globulin heavy-chain gene rearrangements can help to differentiate benign from malignant infiltrates.

There is no standard treatment for tattoo-induced cutaneous pseudolymphomas. Topical application or intralesional injection of corticosteroids is generally attempted, but the results are variable. There has been 1 case in which progression of cutaneous pseudolymphoma to lymphoma was reported [14]. Although this malignant transformation is thought to be extremely rare, surgical excision of the tattoo should be considered whenever possible [13]. In our case, surgical excision would have been inadequate due to the cosmetic outcome and functional morbidity. Therefore, corticosteroid intralesional injections were performed and our patient had a good response.

Cosmetic semipermanent tattoos, usually on the face, are different from permanent tattoos on the trunk or extremities. Semipermanent tattoo dye may be implanted in the epidermis and the upper dermis. The depth of the punctures is between 0.6 and 2.2 $\mathrm{mm}$ [15]. Conventional permanent tattoos, on the other hand, are implanted to a depth of about $3.5 \mathrm{~mm}$, which is below the middermis. The difference in depth between permanent and semipermanent tattoos may influence the prognosis and therapeutic options for tattoo reactions; however, more cases must be studied to verify this.

To the best of our knowledge, this is the first case of a pseudolymphoma induced by a semipermanent tattoo, and also the first pseudolymphoma to occur on the face. Careful dermatologic examination for complications of a semipermanent tattoo is important, given the increased number of such procedures being performed today. 


\section{References}

-1 Ploysangam T, Breneman DL, Mutasim DF: Cutaneous pseudolymphomas. J Am Acad Dermatol 1998;38:877-895.

2 Van Vloten WA, Willemze R: The many faces of lymphocytoma cutis. J Eur Acad Dermatol Venereol 2003;17:3-6.

3 Kahofer P, El Shabrawi-Caelen L, Horn M, Kern T, Smolle J: Pseudolymphoma occurring in a tattoo. Eur J Dermatol 2003;13:209-212.

4 Chave TA, Mortimer NJ, Johnston GA: Simultaneous pseudolymphomatous and lichenoid tattoo reactions triggered by re-tattooing. Clin Exp Dermatol 2004;29:197-199.

5 Sowden JM, Byrne JP, Smith AG, Hiley C, Suarez V, Wagner B, Slater DN: Red tattoo reactions: X-ray microanalysis and patch-test studies. Br J Dermatol 1991;124:576-580.

6 Waldmann I, Vakilzadeh F: Delayed hypersensitivity to red azo dyes in tattoos (in German). Hautarzt 1997;48:666-670.

7 Jäger C, Jappe U: Contact dermatitis to permanent make-up: manifestation of a pre-existing nickel allergy (in German). J Dtsch Dermatol Ges 2005;3:527-529.

8 Jäger C, Hartschuh W, Jappe U: Sunlight-induced granulomatous reaction to permanent lip liner (in German). Hautarzt 2005;56:63-65.

$\checkmark 9$ Kluger N, Phan A, Debarbieux S, Balme B, Thomas L: Skin cancers arising in tattoos: coincidental or not? Dermatology 2008;217:219221.
10 Engel E, Ulrich H, Vasold R, König B, Landthaler M, Süttinger R, Bäumler W: Azo pigments and a basal cell carcinoma at the thumb. Dermatology 2008;216:76-80.

-11 Yazdian-Tehrani H, Shibu MM, Carver NC: Reaction in a red tattoo in the absence of mercury. Br J Plast Surg 2001;54:555-556.

12 Lubach D, Hinz E: A pseudolymphomatous reaction in tattooing (in German). Hautarzt 1986;37:573-575.

13 Muñoz C, Guilabert A, Mascaró JM Jr, Lopez-Lerma I, Vilaplana J: An embossed tattoo. Clin Exp Dermatol 2006;31:309-310.

14 Sangueza OP, Yadav S, White CR Jr, Braziel RM: Evolution of B-cell lymphoma from pseudolymphoma: a multidisciplinary approach using histology, immunohistochemistry, and Southern blot analysis. Am J Dermatopathol 1992;14:408-413.

15 Vassileva S, Hristakieva E: Medical applications of tattooing. Clin Dermatol 2007;25:367-374.

Prof. Sang Wook Son, MD, PhD

Department of Dermatology, Korea University Ansan Hospital

516, Gojan-1-dong, Danwon-ku

Ansan, Kyunggi-do, 425-707 (Korea)

Tel. +82 31412 5180, Fax +82 31412 5184, E-Mail skin4u@korea.ac.kr 